\title{
Role of polymers as crystal growth inhibitors in coprecipitation via solutionenhanced dispersion by supercritical fluids (SEDS) to improve andrographolide dissolution from standardized Andrographis paniculata extract
}

\begin{abstract}
The poor aqueous solubility and dissolution rate of andrographolide in aqueous gastrointestinal fluids often cause low oral bioavailability. In this work, Andrographis paniculata extract containing 16\% andrographolide was coprecipitated with Pluronic F127, Eudragit EPO, and Eudragit L100-55 via solution-enhanced dispersion by supercritical fluids (SEDS) to improve andrographolide dissolution in simulated intestinal fluid $(\mathrm{pH} \mathrm{7.4).} \mathrm{The} \mathrm{SEDS} \mathrm{working}$ parameters were set constant as follows: $150 \mathrm{bar}, 40^{\circ} \mathrm{C}, \mathrm{CO} 2$ flow rate $15 \mathrm{~L} / \mathrm{min}\left(1 \mathrm{bar}, 25^{\circ} \mathrm{C}\right)$, liquid feed flow rate $0.5 \mathrm{~mL} / \mathrm{min}$, and $25 \mathrm{mg} / \mathrm{mL}$ of A. paniculata extract. SEDS coprecipitates formulated with lower Eudragit L100-55:A. paniculata mass ratios exhibited improved andrographolide dissolution in SIF ( $\mathrm{pH}$ 7.4), while SEDS coprecipitates formulated with either Pluronic F127:A. paniculata or Eudragit EPO:A. paniculata at any mass ratio exhibited poorer andrographolide dissolution $(<0.03 \mathrm{mg} / \mathrm{mL}$ released in $90 \mathrm{~min})$ than SEDS-precipitated A. paniculata extract powder $(0.06 \mathrm{mg} / \mathrm{mL}$ released in $90 \mathrm{~min})$. In particular, SEDS coprecipitates formulated with a Eudragit L100-55:A. paniculata mass ratio of 6:25 were found to have the highest andrographolide release and dissolution rate in SIF ( $\mathrm{pH} 7.4)(0.09 \mathrm{mg} / \mathrm{mL}$ released in $30 \mathrm{~min}$ ). SEDS coprecipitation was successful, as indicated by differential scanning calorimetry (DSC), thermogravimetric analysis (TGA) and Fourier-transform infrared (FTIR) analysis.
\end{abstract}

Keyword: Polymer; Crystal growth inhibitor; SEDS coprecipitation; Andrographolide; Dissolution 